\title{
SCOTTY: Fast a priori Structure-based Extraction from Time Series
}

\author{
Athanasios Tsitsipas*, Pascal Schiessle ${ }^{\dagger}$ and Lutz Schubert ${ }^{\ddagger}$ \\ Institute of Information Resource Management \\ Ulm University \\ Germany \\ Email: *athanasios.tsitsipas@uni-ulm.de, ${ }^{\dagger}$ pascal.schiessle@uni-ulm.de, ${ }^{\ddagger} 1$ lutz.schubert@uni-ulm.de
}

\begin{abstract}
The recognition and extraction of data-driven patterns is a challenging task. In vast amounts of data, suitable techniques should prepare data that match the user's intentions. For example, "an increasing daily price of a stock, is generally followed by a sudden fall due to the endorsed profit gains". Such a statement should effortlessly be transferred as input to a data mining task, without the need for an extensive training phase building an extremely complicated model and the usage of threshold-based approaches. We propose a method for extracting lexical representations directly from the raw data, enabling others not directly compatible with real-valued data. We provide evidence that our method is fast and accurate. We use as evaluation a preliminary step for a classification task compared to state-of-the-art classifiers applying it on a publicly available dataset.
\end{abstract}

Index Terms-time series representation, shapeoids, symbolic aggregate approximation, shape representation

\section{INTRODUCTION}

In the Big Data era, the generation of massive amounts of data over time is evident. Data are collected in application domains ranging from health (ECG signals), finance (stocks, sales), industry (wind generation), to sensors recording time series data, to name a few. Typically, a time series collects data points indexed in equally spaced sequential measurements over time [1]. Different data mining tasks from a time series arise from our natural reification incentive to envisage data shapes in them. Humans reckon on various complex biological modalities to attain such tasks. We can perceive the notion of a shape, even from fluctuated data, and almost instantly find similarities between different patterns in various time scales.

Identifying shapes or patterns in line charts is a vital part of data exploration. Domain experts intend to make sense of their datasets, acquire new insights, and collectively examine their hypotheses against the data [2]. For example, financial analysts inspect stock prices in the form of patterns, which are usually described in natural language [3], to understand and predict the market; biologists examine patterns of gene expressions over time to study biological processes [4]; clinical data analysts may predict health events of their patients using trends in their health indicators [5] (e.g., temperature, blood pressure etc.).

These domain experts heavily rely on visual analytics tools offering expressive and flexible ways to express the patterns of interest [6], [7]. Most tools expect the users to sketch a complete trendline or express it using regular expressions and threshold definitions, making the task even more tedious. Although such tools have taken over some of the burdens of expressing users' intentions, they are still prone to searching in constrained ways. They are rigid in how they assess pattern matching. In addition, traditional time series data mining techniques [8] also have the ability to search for patterns in time series via sub-/sequence pattern matching using similarity search. However, they require heavy preprocessing of the time series (e.g., alignment [9]), limiting the time-sensitive use cases. All of the above approaches share a common step at their core; the time series representation.

According to a recent study [2], the subjects were called to describe time series sequences using an English sentence. They often described complex patterns using a combination of multiple simple ones, using words such as "increasing", "stable", or "falling". Examples of such complex patterns are "gene expressions that rise and then become stable" [2], or "a period of low telephone call activity is usually followed by a sharp rise in call volume" [10]. Many other research works have described basic patterns in natural language to express the trendline [10]-[12]. Our emphasis is how humans can observe and describe a time series by locking to some points of interest to perceive a general shape. They are not restricted by the temporary fluctuations or existing noise (i.e., high dimensionality of data).

We lend inspiration from the above, and we propose a set of apriori ${ }^{1}$ lexical shape-like primitives, named shapeoids, coupled with their trend directions to describe and extract them from a time series as a pre-processing step for input to any data mining task (e. g., classification, query by content, clustering, rule discovery). Additionally, with the shapeoids we enable other methods that are not directly compatible with real-valued data [13], such as symbolic logic-based reasoning [14], suffix trees, or many algorithms from the domain of natural language processing. In addition, we have developed a framework named SCOTTY for extracting them without any training data while making it robust to noise. We offer this "morphable" approach as a time series representation in a preprocessing

\footnotetext{
${ }^{1}$ According to the Oxford Dictionary, the word a priori means "Relating to or denoting reasoning or knowledge which proceeds from theoretical deduction rather than from observation or experience". We use innate knowledge to describe a trendline (e.g., the mental model of a flat line ).
} 
step for a fast similarity measure ${ }^{2}$ and its description in natural language constructs. We evaluate SCOTTY over a "declarative" classification task, using the discovered shapeoids as simple shapes to form more complex shapes in a synthetic dataset from the UCR Time Series Archive ${ }^{3}$.

The rest of the paper is organised as follows. In Section II we give an overview of methods moving from real-valued data to symbols and then in Section II-C discuss related work. Next, in Section III we describe the contributions, presenting our framework SCOTTY and its internal mechanisms, while in Section IV we evaluate it. Finally, in Section V we summarise the work and propose future work directions.

\section{BACKGROUND}

In this section, we describe the concept of time series representation and discretization. Next, we present a well-known technique for symbolic representation method called Symbolic Aggregate Approximation (SAX). SAX contains basic steps, first the approximation by reducing the high-dimensionality of the time series using a method called Piecewise Aggregate Approximation (PAA), which then is discretized to symbols via quantisation. This reduces the noise of the time series while offering a symbolic representation as a result.

\section{A. From real-valued data to symbols}

Due to the high-dimensionality in time series data, operating directly on the raw time series becomes computationally expensive. Instead, discriminant features are extracted and processed. Such techniques fall under the umbrella of dimensionality reduction as part of the time series representation; their main focus is to reduce the length of a time series drastically. The profit gains in terms of storage, low computational costs and noise removal are significant [1]. Agrawal et al. in their pioneering work, suggested an approximated representation for time series [15]. Since then, many works have emerged proposing various approaches offering different trade-offs. A valid dimensionality reduction technique (or approximation) will transfer some distinct data points in space, which are substantially less than the original data, and at the same time do not alter the original data curve [16]. Time series representations belong to two categories: numeric and symbolic. Numeric reduction techniques draw inspiration from the field of signal processing, using filters and other compression methods. Some examples are Discrete Fourier Transform (DFT) [15], [17] based on Fourier coefficients, Discrete Wavelet Transform [18] based on Wavelet coefficients, Piecewise Aggregate Approximation [19] and Adaptive Piecewise Constant Approximation (APCA) [20] based on mean values, to name a few.

On the other hand, symbolic reduction techniques boil down to symbols, which are essentially character strings. Using symbolic representations, researchers were able to index huge datasets in a memory-efficient manner [21]. One reason that symbolic representations perform so effectively is the

\footnotetext{
${ }^{2}$ We use this term to denote the "earliness" of the result.

${ }^{3} \mathrm{https}: / /$ www.cs.ucr.edu/ eamonn/time_series_data_2018/
}
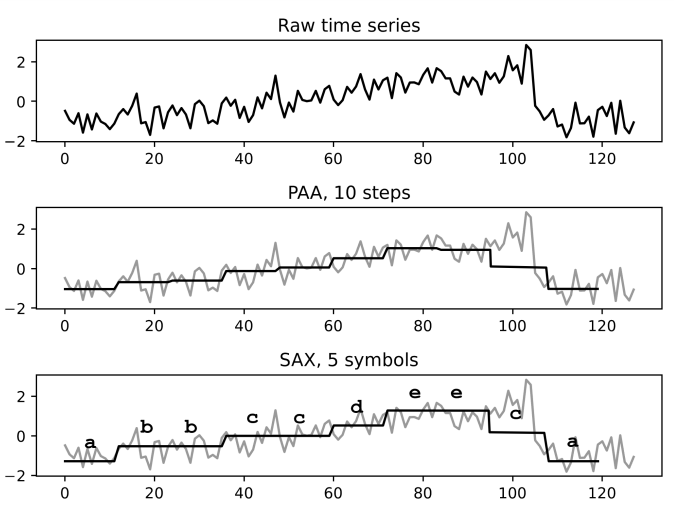

Figure 1: The dimensionality reduction of a raw time series using ten PAA steps (i.e., partitions) and selecting letters for SAX from an alphabet of five. The final graph also contains the concrete discretisation of the assigned letters.

approximation part, which is applied to map a time series to its reduced version in a vector of real values. Subsequently, each real value in the vector, via a quantization procedure (using an alphabet size), maps to an interpretable character symbol.

Furthermore, we discuss two prominent symbolic representations of time series: the Symbolic Aggregate Approximation (SAX) [22] and the Symbolic Fourier Approximation (SFA) [23]. SAX approximates the time series using PAA and presumes the PAA values to follow a Gaussian distribution. Next, SAX discretizes the real values to symbols according to equal-sized partitions in the Gaussian curve offered by breakpoints. On the other hand, SFA uses a DFT method to approximate a time series, which is established in the community of signal processing and acts as a noise removal filter. To the degree of the quantisation step, SFA uses a Multiple Coefficient Binning (MCB) method, generating a lookup table from various DFT approximations of training data. ScOTTY bases its implementation on SAX because it is agnostic to the underlying use case, assuming no training data is available. Therefore, the quantisation step of SFA would be not possible. A recent work [24] used an ensemble of SAX and SFA interchangeably for a classification task, but they couple their approach with learning for achieving top-notch accuracy results. Nevertheless, it simply does not conform with our notions when we were designing SCOTTY. We envision, a fast and efficient way to move from the data to human-relatable representations.

\section{B. SAX}

Since our work is based on SAX, we briefly review the method. We refer the reader to [22] for the original description of the method. For concreteness, we begin with the definition of the time series. A raw time series $T$ consists of a set of real numbers $r \in \mathbb{R}$, which are sampled over time: $T=$ $\left\{t_{1}, t_{2}, \ldots, t_{r}\right\}$. The main result of SAX is the transformation of a time series to a sequence of symbolic letters forming the so-called SAX word of the time series. The $T$ in SAX is 
Table I: A statistical lookup table containing the breakpoints splitting the Gaussian distribution into several equiprobable partitions, depending on the alphabet size.

\begin{tabular}{ccccccc}
\hline & $\mathbf{3}$ & $\mathbf{4}$ & $\mathbf{5}$ & $\mathbf{6}$ & $\mathbf{7}$ & $\mathbf{8}$ \\
\hline$\beta_{1}$ & -0.43 & -0.67 & -0.84 & -0.97 & -1.07 & -1.15 \\
$\beta_{2}$ & 0.43 & 0 & -0.25 & -0.43 & -0.57 & -0.67 \\
$\beta_{3}$ & & 0.67 & 0.25 & 0 & -0.18 & -0.32 \\
$\beta_{4}$ & & & 0.84 & 0.43 & 0.18 & 0 \\
$\beta_{5}$ & & & & 0.97 & 0.57 & 0.32 \\
$\beta_{6}$ & & & & & 1.07 & 0.67 \\
$\beta_{7}$ & & & & & & 1.15 \\
\hline
\end{tabular}

finite. As such, the length of a time series scopes its size. The static nature of $T$ is a fundamental characteristic of performing the initial normalisation step, as we will showcase. As such, the normalised time series set is $\hat{T}=\left\{\hat{t}_{1}, \hat{t}_{2}, \ldots, \hat{t}_{l}\right\}$, with a mean of zero and a standard deviation of one. The next step of dimensionality reduction using the PAA method, over $\hat{T}$, is defined as $\bar{T}$ containing $\omega$ equal partitions $t_{i}$ :

$$
\bar{t}_{i}=\frac{\omega}{n} \sum_{l=\frac{n}{\omega}(i-1)+1}^{\frac{n}{\omega} i} \hat{t}_{l}
$$

To reduce the initial normalised time series from its current dimension $n$ (i.e., its length) to another dimension, the data is split into equal-sized partitions $\omega$; each partition represents the mean value of the data falling into it.

The final discretisation step is the conversion of the numerical set $\bar{T}$ to a string-based SAX word $\mathcal{W}$ of length $\omega$. The number of letters $\alpha$ constitutes the level of smoothness of the initial time series. The higher the value, the more features are visible. To determine the distribution of letters, SAX uses the partitions in $\bar{T}$ and assigns the letters based on breakpoints $B=\left\{\beta_{1}, \ldots, \beta_{\alpha-1}\right\}$. In the Gaussian distribution $\mathcal{N}(0,1)$, the probabilities of a split region $\left[\beta_{i}, \beta_{i+1}\right]$ are equal to $1 / \alpha$, with $\beta_{0}=-\infty$ and $\beta_{\alpha}=+\infty$. The lower partition assigns to the letter ' $a$ ', the next passing a breakpoint, to ' $b$ ', and so on. Table I enumerates the breakpoints for the number of characters in the alphabet $A$.

While SAX gains popularity for its simplicity and efficiency endures considerable information loss. It removes trends and shapes information in a time series as PAA is a preceding step. The data distribution changes and results in a shrinking form. In [25], the authors state that the SAX method does not guarantee equally probably symbols, affecting the final discretisation negatively. Nonetheless, SAX has a central role in our approach. We provide evidence that it preserves the shape and trend information sufficiently. In fact, we give an overview of how previous works enhance SAX for the trend information and which research endeavours relate to our approach; to extract a discrete data-driven shape-like symbolic representation from a time series.

\section{Related Work}

Recent works alleviate the information loss of SAX by employing additional information in its definition. The original
SAX is amalgamated with altered lookup tables and slopes by regression. Extended SAX (ESAX) [26] mingles the original definition with the minimum and maximum points for each PAA partition, associated with their SAX symbols while preserving their occurrence order. This defines an abstract shape used for information retrieval. Although the approach is in line with ours, it adds a burden on storing the representation as it becomes three times larger. The same concept could be achievable via raising the number of PAA segments resulting in more fine-grained divisions. In [27], Esmael et al. introduce symbols for describing the time series trend for each partition, using the values $\mathrm{U}$ (up), D (down), and S (straight). They retrieve the trend approximations via linear regression and quantize the line slope. This alters the SAX definition completely and raises the storage factor by two. Zalewski et al. in their paper, quantize the slope by representing it as a difference between two successive partitions in the time series. They translate the difference values into $k$ classes, determine the related centroids and calculate the slope degree. The main drawback of this approach is the loss of simplicity and readability representing the time series using the degrees of the slope formation. Although the authors are in the same direction concerning the slope direction, we remain simplistic and intuitive at the same time (cf. Section III). Malinowski et al. use the slope values and the mean values of a linear regression task to quantize the partitions of a time series, resulting in subsequence slope "directions", while the compression ratio of the time series was the same as in original SAX. Finally, the works in [28], [29] extract the trend information, without linear regression, and append it into the basic SAX. They provide sufficient evidence that they improve classification tasks and preserve interpretability. However, they still add additional storage complexity.

Symbolic representation has been increasingly popular in research areas of the query by content. Agrawal et al. define basic shape patterns such as, "up", "down", "stable" and create a query language named Shape Definition Language (SDL) [11] for searching these patterns based on their occurrences. However, this approach operates on raw data. It is susceptible to noise, while it is unclear how they move from the numeric data to the proposed symbolic classes. In the context of similarity search for feature extraction, there are two main categories [30]: the shape-based and the structurebased. Shape-based similarity uses distance measures on realvalued time series (e.g., Euclidean Distance or Dynamic Time Warping ). Structure-based approaches use higher-level constructs from a time series to make various existing data mining algorithms applicable; for more information, we point the reader to this survey [1]. They employ representations derived from either dimensionality reductions techniques or the relatively modern concept of shapelets [31]; subsequences that are candidates for describing a class. The basic difference is the amount of training data a shapelet requires to be identified as a representative class. In contrast, in our approach, we can be agnostic to a training phase. We can use samples of data only for optimisation purposes of the input parameters 
SCOTTY requires.

SCOTTY introduces a primitive concept named shapeoid, which describes the time series curve using lexical representations (e.g., Flat, upward ANGLE, downward HoRn etc.). They raise the interpretability of a time series without introducing any additional burden in the underlying technique of SAX. Using a segmentation technique and string-based methods for their extraction (instead of a similarity measure), we lift the representation from the obscure symbolic letter sequence of SAX to a representation in natural language.

\section{THE SCOTTY FRAMEWORK}

In this section, we present our structure-based representation framework named SCOTTY. It heavily relies on SAX as it operates directly on the SAX word for extracting the indigenous primitives in the framework (i. e., shapeoids). We begin the section with some useful notation. Next, we describe the individual shapeoids, and finally, we present our approach to extracting them.

\section{A. Notation}

Definition 1. SAX Word: A SAX word $\mathcal{W}$ is a sequence of characters $w_{0}, w_{1}, \ldots, w_{\omega}$ from the alphabet $|A|=26$, with length $\omega$ reflecting the PAA partitions.

The definition applies for a time series $T$, but also for a local view in a SAX word from $T$.

Definition 2. Subsequence: Given a word $\mathcal{W}$, a subsequence $\mathcal{S}$ of $\mathcal{W}$, is a sampling of length $\sigma \leq \omega$ of successive positions in $\mathcal{W}$, that is $w_{p}, \ldots, w_{p+\sigma-1}$ for $1 \leq p \leq \omega-\sigma+1$.

SCOTTY characterizes each subsequence instead of the whole time series, as we will elaborate below.

Definition 3. Sliding Window: Given a $\mathcal{W}$ of length $\omega$ and a defined subsequence $\mathcal{S}$ length of $\sigma$ with a sliding period $\rho$, all possible subsequences can be extracted by sliding a window of size $\omega$ across $\mathcal{W}$ and considering each subsequence $\mathcal{S}_{\rho}^{\sigma}$ of $\mathcal{W}$. Here the superscript $\sigma$ is the length of the subsequence, and subscript $\rho$ indicates the initial position of the window after sliding it through $\mathcal{W}$.

In modern data processing frameworks, windowing techniques have a central role. Following Definition 3., it is a standard technique to select data based on a fixed amount of time. It has also been used for accommodating the need of finding motifs and discords in a time series [32]. Based on it and combining it with SAX, numerous works produced significant results, most notably dictionary-based classifiers such as BOP [30] and SAX-VSM [33]. Such works resemble our approach for extracting patterns. However, we do not cover a data mining task explicitly as SCOTTY is a purely preprocessing framework turning a time series into a series of interpretable lexical representations.

Definition 4. Multiset of words: The multiset of all subsequences of length $\sigma$ extracted from $\mathcal{W}$ is defined as $S_{\mathcal{W}}^{\sigma}=\left\langle S_{\rho}^{\sigma}\right.$ of $\mathcal{W}$, for $\left.1 \leq \rho \leq \omega-\sigma+1\right\rangle$.

We put forward that a time series translates to a multiset of words as an abstract symbolic representation of its curve.
Definition 5. Shapeoid: Let $s \in S_{\mathcal{W}}$ be a word of length $\sigma$. The local word $s$ forms a shapeoid if and only if it is a triad, i. e., $|s|=3$.

The shapeoid is a lexical characterisation of a word as interpretable applied to any domain. We define four individual shapeoids ANGLE, Hop, HORN and Flat. On top of them, there exist also orientation classes or trends, named as upward and downward. The step of dimensionality reduction in SAX and its discretisation process lifts the computation from the numerical real-valued elements to symbolic interrelations.

\section{B. Shapeoid Primitives}

Next, we will elaborate on the definitions of the individual lexical shapeoids (i. e., ANGLE, Hop, Horn, Flat) and their trends (upward and downward). The shapeoid extraction is a string-based operation over the global SAX word of a time series after applying a sliding window (cf. Section III-C). The transformation of the local SAX words aids the human-related interpretability of the time series. Therefore, we standardise these definitions as a minimal feature set to translate a time series to natural language constructs. The most common application of shapes and patterns is in financial technical analysis; using domain-dependent identification of abstract shape occurrences, such as the inverse head-and-shoulders pattern or the cup-with-a-handle to describe a possible uptrend of a stock [34]. We provide an overview of our patterns - the shapeoids:

ANGLe: An Angle is a gradual and continuous increase or decrease in the original time series $T$. Some works usually describe this as a slope [2], [35], [36].

Hop: A HOP describes a distinctive phase shift in the original time series $T$, leading to an overall visible change. The pattern resembles the ANGLE; however, a HOP has a more drastic effect in the trendline, which changes it radically either upwards or downwards. Other words found in the pattern extraction literature for this shapeoidis the jump [37] and sharp [10], [38].

HORN: The HORN is an ephemeral increase or decrease in the time series $T$. Thus, a short-term effect fades quickly, as the end of the pattern returns almost to the initial point. It is a rather frequent pattern that appears in research works with other words, such as a spike [35], and a peak [16]; a downward HORN is also described as a valley in the financial domain [34].

FLAT: The Flat is an almost sturdy line with small variations in the curve. It is a rather common pattern found in many time series, and it identifies as the "calmness before the storm". Many works refer to this pattern as the stable [2] pattern or the horizon [37] pattern.

As mentioned above, the transformation of a local SAX word to a shapeoid is a purely string-based computation. We do not use any similarity measure (e.g., Euclidean distance), and we avoid the need for parametric thresholds in our approach to extract the shapeoid in focus. For our notation (cf. III-A), the local word is a triad of characters from the given SAX alphabet. In our previous paper [39], we formally 


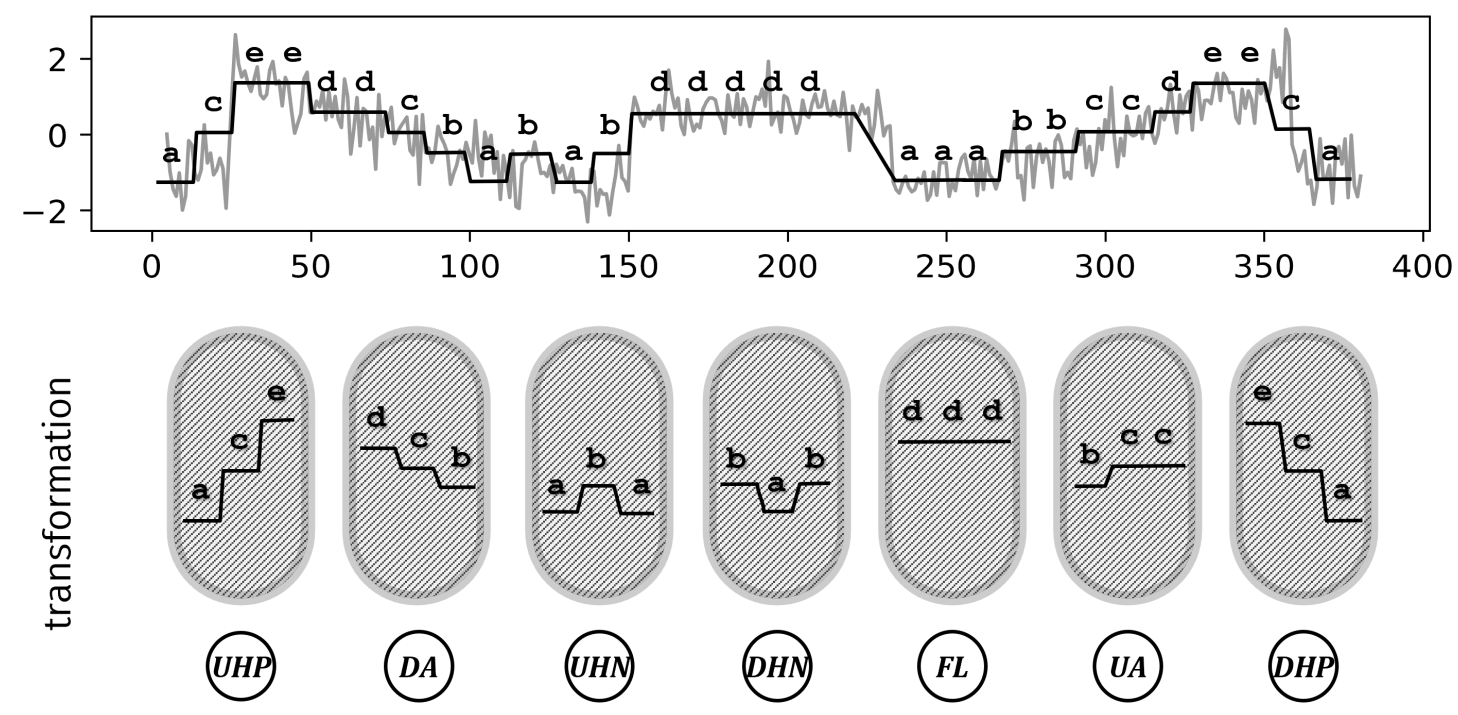

Figure 2: Transforming the local SAX words in the sliding window over the global SAX word on top; uses the merged raw time series data from all three classes in the CBF dataset. The merged time series contains all the lexical shapeoid primitives; we list local SAX words for the shapeoids. Algorithm 1 contains the pseudocode of the shapeoid transformation. Abbreviations: (UHP) upward HoP, (DA) downward ANGLE, (UHN) upward HORN, (DHN) downward HoRN, (FL) FLAT, (UA) upward ANGLE, (DHP) downward HoP.

provide evidence about the correctness and the complexity of the shapeoid transformation.

The Algorithm 1 describes in pseudocode the shapeoid transformation function and requires the current window slide of the SAX word to proceed with the comparison. The COMPARE function is a simple string comparator, which returns the differences between characters as an integer (line 5-6). The variables $w_{0}, w_{1}, w_{2}$ contain the characters of the SAX word at the respective indexes. For the FLAT and the HORN, lines 7-9 assist the reader to grasp their meaning. The interesting point in the transformation is the interchangeable situation between an ANGLE and a HOP. The transformation conditions for HoP resemble the ones from ANGLE; however, we do not opt for a successive change in the characters of the word, but an instantaneous change which translates to omitting the successive character in the alphabet $A$. Therefore, the characterisation of the HoP is evident from the distance of the letters in the SAX word. For comprehensibility purposes, we illustrate in Figure 2 the shapeoids found in a combined time series all the classes in the Cylinder-Bell-Funnel dataset from UCR Time Series Archive.

\section{Multiset of shapeoids in a time series}

SCOTTY executes the SAX method on the given time series, and the result is a global SAX word. From this point, we will elaborate on how the algorithmic procedure in SCOTTY operates on a SAX word to extract the various shapeoids. The upper goal is to extract a multiset of SAX words, yielding a "vocabulary" of strings for our time series. To achieve this, we use a pre-defined sliding window of length three and an offset of one. Each subsequence corresponds to the time series

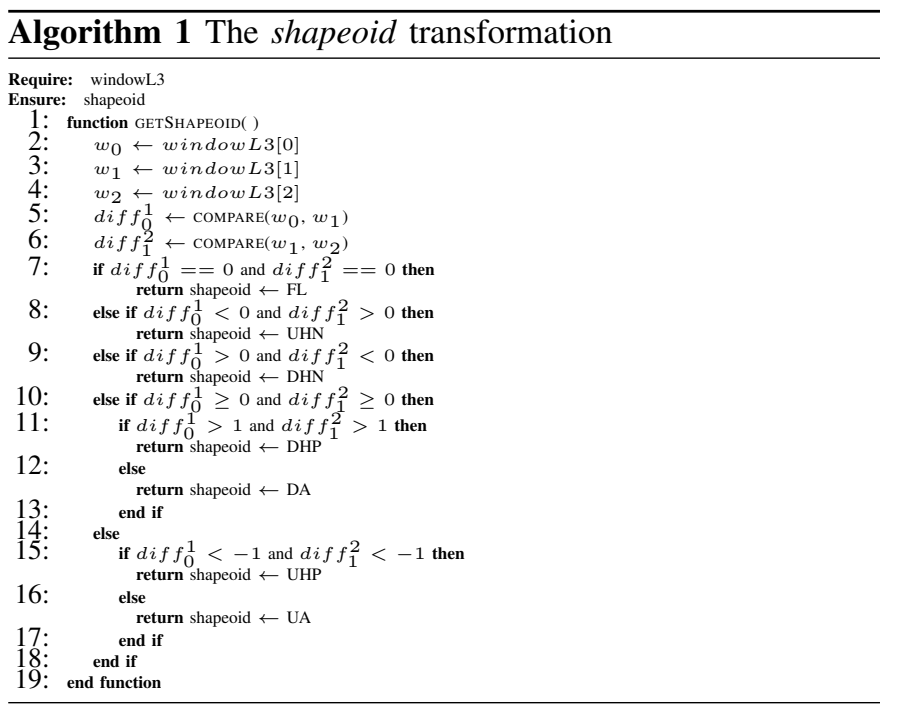

because SAX normalizes the time series with a mean value of zero and a standard deviation of one. Consequently, we get a multiset ${ }^{4}$ of local SAX words. As an example, consider the following sequence of local SAX words extracted from the SAX word (abbccdeeca) in Figure 1, while applying the sliding window:

$$
S_{\mathcal{W}}^{\sigma}=\mathrm{abb}, \mathrm{bbc}, \mathrm{bcc}, \mathrm{ccd}, \mathrm{cde}, \text { dee, eec, eca }
$$

The sequence contains local SAX words, ready for their shapeoid transformation. Internally, SCOTTYstores the shapeoidobject coupled with useful information for any data mining task to follow. The interim representation of a local

\footnotetext{
${ }^{4}$ We use the term mathematically precise.
} 
SAX word contains a triad of SAX letters. Each of the letters is coupled with their index in the dimensional reduced form. During the transformation phase (cf. Algorithm 1), the shapeoid object contains the primitive type (cf. Section III-B), the SAX word (which was transformed from), and the start/end index of the shapeoid (using the edge letters in the word). The transformation algorithm is agnostic to the number of the SAX alphabet letters. Hence, many local SAX words could result in the same shapeoid.

$$
S^{\prime}=\text { UA, UA, UA, UA, UA, UA, DHP, DHP }
$$

As Lin et al. recognised in [13], given a subsequence $S_{i}$, it may be likely to be analogous to the proximal subsequences $S_{i-1}$ and $S_{i+1}$ (i.e., those starting to one step on the left and one step on the right of $S_{i}$ ). After their transformation to shapeoids, we may see the same shapeoid in successive window slides. In SCOTTY, to avoid storing many occurrences of the same shapeoid, we apply numerosity reduction [30]. The numerosity reduction, is more efficient in SсоTTY, as in the approach from Lin et al. for example, different sequential ;etter triads could result in the same shapeoid. We lock the first and last consecutive occurrence and record it once. At the same time, we store the edge letters' index numbers to grasp the full extent of duration.

Applying the numerosity reduction to $S^{\prime}$, we get a cleaned view of the shapeoids. However, in contrast to the "bag-ofwords" approaches, we do not count the duplicates in the multiset for generating a histogram. Instead, the first occurrence of the shapeoid is registered, and we skip all the duplicates until a new shapeoid is exposed. However, we keep the last duplicate (before the new shapeoid), and we register the indexes of the edge letters in the constituting identical shapeoids. Thus, we end up with a duration of the registered shapeoid. In Figure 3, we illustrate the above procedure more elegantly.

\section{ExPERIMENTAL EVALUATION}

SCOTTY acts as a preprocessing step following a dictionarybased time series representation and discretisation approach. The resulting shapeoids are able to compose into more complex shapes/classes, expressing their relationships via sequential operators or other methods (e.g., from the domain of pattern mining). We choose to evaluate the accuracy and efficiency of SCOTTY via a classification task for the CylinderBell-Funnel (CBF) dataset from the UCR Archive. The CBF dataset has been widely used as a benchmark to evaluate time series classification algorithms. In the following, we discuss the setup and the results following our evaluation approach. Our results are highly prominent under specific conditions, which we discuss thoroughly in the paper.

\section{A. Selecting the dataset}

We start by introducing the designated dataset. The CBF dataset is a widely selected benchmark. It contains two separate dataset files; one for training (30 time series) and one for testing purposes (900 time series). Each time series is univariate, sampled at equal intervals and has a length of one hundred

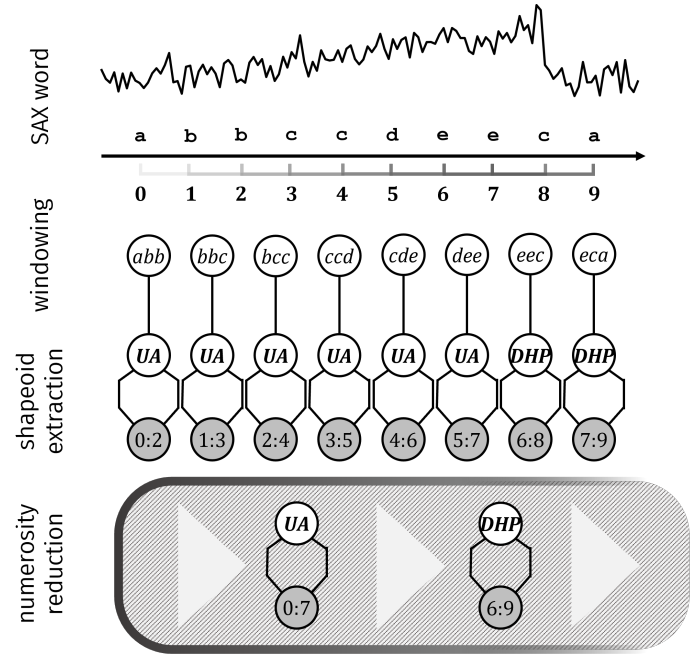

Figure 3: An elaborated view of the interim representations in SCOTTY. The numerosity reduction phase unfolds over the transformed shapeoids in the time series and not in the "bagof-words" representation (i. e., before the shapeoid transformation).

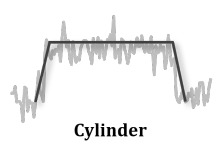

Cylinder

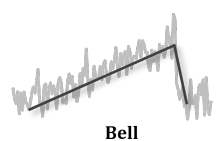

Bell

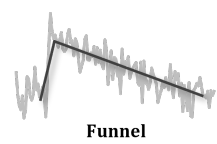

Funnel
Figure 4: A recognisable view of the outlined core shapes in the graphs of a Cylinder, a Bell and a Funnel time series.

twenty-eight. Overall the classifier feeds SCOTTY with one hundred fifteen thousand two hundred data points. The reason for selecting this dataset to evaluate our framework is an obvious asset; it contains shapes visible with the naked eye if plotted in a graph. A human can observe the time series and realize that she can express the described shapes with a sequence of simpler other shapes. In Figure 4, we illustrate the forming patterns we derive by gazing at the plotted trendline of each class.

The following descriptions align perfectly with the CBF design. We begin with the cylinder class. There is a clear upward sudden change in the data ( $\simeq$ upward HoP). The data oscillate over the same horizon ( $\simeq$ FLAT), and then there is another sudden drop in the data $(\simeq$ downward HoP). Next, for the bell class, there is an increasing slope in the data $(\simeq$ upward ANGLE) followed by a sudden drop ( $\simeq$ downward HOP). Finally, the funnel class begins with a sudden increase ( $\simeq$ upward HOP) and continuous gradually downwards $(\simeq$ downward ANGLE). We can perceptually extract these descriptions of what we see and translate them to shapeoids without calculating any complex similarity measure. Therefore, the classifier will use the temporal findings of the shapeoids to classify each time series. We do not use the traditional classification methods, including a similarity measure as an internal task, but a more "declarative" classifier for the pattern mining. 


\section{B. Evaluation Strategy}

The evaluation strategy is two-fold. Initially, we plan to calculate and discuss various performance measures of SCOTTY classifying the predetermined shapeoids from the $\mathrm{CBF}$ dataset. A forthcoming requirement for this is the custom classifier built upon SCOTTY and has a pattern matching flavour in its definition. We define the shape of the trendlines from the given classes (i.e., cylinder, bell, funnel). We provide evidence that a data mining task (i. e., classification) is implemented with the help of SCOTTY. The custom classifier is implemented using three implementation designs:

Strict: We envisage a perfect world where the identification of the aforementioned happens by a sequential matching of the class definitions (cf. Section IV-A). There may be some noisy data that tamper with the initial definition of the shapes and do not evaluate precisely. To tackle this, we introduce the next scenario based on a well-known time series classification technique: the Perceptually Important Points.

Perceptually Important Points (PIP): The PIP is an elegant method to retain the key turning points in a time series. It was first introduced by Chung et al. [40] and is widely used in pattern recognition methods [35]. We use a similar method à la "PIP", but instead of finding salient points, we match the occurrences of shapeoids in the given CBF shape definition by focusing on it and skipping subsequent "noisy" shapeoids which do not match with the given definition. Whilst fulfilling each CBF definition temporally, we do not do turnarounds in the classification algorithm. However, we sequentially look for the non-matched shapeoid at the given index. For example, we may get locked in the case where a shapeoid is nowhere to be found, not even in subsequent identified shapeoids.

Optimising the Shape Definitions: In this scenario, we use the ANGLE and HoP shapeoids interchangeably for the CBF classes. The reason is that we may not match one of the two shapeoids. However, we have the background knowledge that some additional noise in the data may result in a "noise" in the dimensionally reduced data and interfere with the declarative classification task. For example, a time series in the test data could look like Hop but the increase is not that sudden and will be recognised as an ANGLE.

Overall, the above scenarios for our declarative classifier are essential to show the different performance measures. We substantiate the importance of using structure-based representations, such as the shapeoids, because they enhance the way of how humans perceive forming shapes in charts [3].

\section{Setup}

We evaluate SCOTTY in a classification task with different implementation designs and compare the results. SCOTTY and the custom classifier were implemented in Java using OpenJDK 11.0.6. Internally, SCOTTYemploys an open-source Java implementation ${ }^{5}$ of SAX. All the raw numbers from the evaluation and source codes are publicly available under the Open

\footnotetext{
${ }^{5}$ https://github.com/jMotif/SAX
}

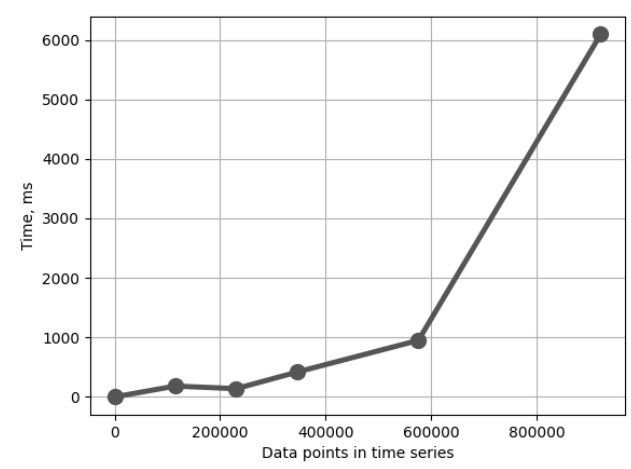

Figure 5: SCOTTY's run time (in milliseconds) with an asymptotic increase in the time series number.

Science Framework project ${ }^{6}$. All experiments were performed on a shared memory machine running Linux on an AMD Ryzen 5800X with eight cores (sixteen threads) and thirtytwo GB of RAM using a 970 Plus NVMe SSD for storage.

\section{Results}

The evaluation results follow the evaluation strategy we described above. Initially, we examine the efficiency of SCOTTY to extract the defined shapeoids for the classification task. We select a prominent combination of parameters. We present its efficiency and the run time duration with an asymptotic increase in the time series number. Finally, we discuss the results from an unconventional classification task, which employs declarative specifications of the shapes/classes for identification. We compare the different implementation designs of our classifier with well-known structure-based classification techniques; the Shapelets [31], the Bag-of-Patterns (BOP) [30] and the BOSS classifier [41].

SCOTTY: It has an uncommon evaluation method. It is evaluated as a preprocessing step in a custom time series classification task (cf. Section IV-B). Since SCOTTY outputs local lexical representations of a time series, the only option to evaluate its efficiency and accuracy is a human-related task. However, since publicly available datasets (e.g., CBF, Mallat etc.) were simulated to output raw real-valued time series (e.g., the CBF dataset) for a specific class of data, we can profit from their definitions. Therefore, the ground truth (i.e., labelled data) is interwoven with the underlying shape definition in the given class (cf. Section IV-A).

As part of our evaluation, we run an experiment on how well SCOTTY scales in the presence of almost a million data points. We did six runs, and we isolated the run time of ScOTTY. The time series increase bases on the nine hundred time series from the CBF dataset, following a Fibonacci growth rate (e.g., zero, one, one, three, five, eight etc.). In Figure 5, someone can observe how good SCOTTY scales in the presence of huge amounts of data. With seven thousand two hundred time

\footnotetext{
${ }^{6}$ https://osf.io/d5f3c/
} 
series (containing almost a milion data points), SCOTTY takes approximately six seconds to process them. For the sake of readability, we did not increase the time series number using the next Fibonacci number (i. e., 13), as the resulting execution time would make the previous readings in the plot unreadable. The total run time (approximately one and a half million data points) was three minutes and seven seconds. The time and space complexity are both $\mathrm{O}(\mathrm{n})$.

In Figure 6, we show the efficiency of SCOTTY recognising the shapeoids defined in the classifier (cf. Section IV-A). The figure contains five plots, one for each specified shapeoid. Each plot contains performance measures over eight runs, while increasing the cardinality of the SAX alphabet as a SCOTTY parameter. The other parameter (i.e., PAA partitions) remains stable to the number ten. The classifier runs with the implementation of PIP. We do not use the optimisation (i. e., interchanging the ANGLE and HoP dynamically) in the shape/class definitions since this will tamper the real efficiency of SCOTTY in the context of the classification task. The accuracy of SCOTTY is irrelevant at this step since we cater for its real efficiency. We observe that the Recall is almost around the perfect score while using different SAX alphabet letters. The Figure 6e indicates lower scores for the downward HoP, as it consists the last part in two shapes definitions (Cylinder and Bell). Using the PIP implementation of the classifier, there might be a drop at the ending part of the shape definition. The factors leading to this are its inability to find a preceding shapeoid due to the nature of the given time series or a weakly labelled time series [9].

Declarative Classifier: The declarative classifier was initially a way to evaluate SCOTTY, its preprocessing step for the time series representation. However, it became a very prominent artefact competing with state-of-the-art structure-based classifiers. As such, we provide evidence for the accuracy and efficiency of SCOTTY in the context of data mining.

Figure 7 contains an illustration of the classifier's error rate for the different implementation designs while varying the SCOTTY parameters with the following schema: for every $n$ SAX letters, the PAA partitions are two times $n$. It is a linear growth while maintaining the size of the partitions in low levels. The results show lower error rates for PIP with, or without, the optimisation when the cardinality in the parameters increase. At the same time, there are higher error rates for the STRICT method and the optimised STRICT (cf. Section IV-B). The STRICT method has an accuracy of almost fifty percent, which is a shallow result for the given task. Optimising the STRICT method raises the accuracy to seventytwo percent. When the PAA partitions start to increase, the STRICT methods cannot cope with the mining task due to the inability to find the whole shape definition in sequence. On the other hand, the PIP method has relatively good results, with an intermediate combination of parameters of fixed SAX letters and ten PAA partitions. This parameter combination is a salient point, as the classification accuracy will raise in their further increase. However, the underlying idea behind the classifier's design will lose its credibility. The more the
Table II: Classification accuracy and running time between Shapelets, Fast Shapelets, BOP, BOSS, SCOTTY STRICT, SCOTTY STRICT + OPT, SCOTTY PIP, SCOTTY PIP+OPT.

\begin{tabular}{lcr}
\hline Classifier & Accuracy & Duration (s) \\
\hline Ultra Fast Shapelets [42] & 0.886 & 4.7 \\
Fast Shapelets [17] & 0.947 & 7 \\
1-NN BOSS VS [43] & $\mathbf{0 . 9 9 8}$ & 0.48 \\
STRICT & 0.480 & 0.232 \\
STRICT+OPT & 0.729 & 0.234 \\
PIP & 0.903 & 0.254 \\
PIP+OPT & 0.983 & $\mathbf{0 . 2 5 5}$ \\
\hline
\end{tabular}

numbers increase, the more coarse the dimensionality reduced time series becomes. This leads to more shapeoids findings, which were meant to be removed as noise. Therefore, the shape definition from the dataset will be found eventually with a perfect score. This implementation design aimed to skip imminent possible noise right after the previous element in the shape definition, where after one or two steps to find the desired shapeoid.

In addition, we include the ratio between the run time of SCOTTY and the classification (cf. Figure 80 ). The recorded times average over three individual tries per dataset from the asymptotic growth rate in the number of the enclosed time series (cf. Section IV-D). We observe a sudden increase in the ratio, occupying most of the processing time in the overall classification. This is due to a development mistake during the built of the classifier (not internally in SCOTTY), which made its time complexity solvable in $\mathrm{O}\left(n^{3}\right)$. However, the classifier works surprisingly good, despite its underlying computational issue.

Furthermore, in Table II we list a comparison between stateof-the-art classifiers according to their accuracy and prediction duration. The best classifier for the $\mathrm{CBF}$ dataset, according to the results and the official website hosting the dataset is a classifier based on the BOSS model. Although our classifier achieves remarkable results (e.g., PIP + OPT), has another advantage: its rapidness with a relatively good result compared to the state-of-the-art classifier. This will assist further timesensitive application, which require quickly lexical representations from the data. We need to state that it is not the paper's purpose to develop an efficient and accurate classifier but to provide evidence that our lexical primitives (i.e., shapeoids) are appropriate as an input to methods that are not directly compatible with real-valued data. For example, developing the declarative-based classifier in the context of the evaluation.

\section{Conclusion \& Discussion}

The paper introduces the shapeoids; a set of primitive datadriven lexical constructs as part of a time series representation framework, named SCOTTY. The transformation of a time series to lexical representations will assist methods that cannot operate on numerical data due to their processing nature. In addition, the use cases that we foresee for our approach are similar to the chart patterns in the stock market analysis or 


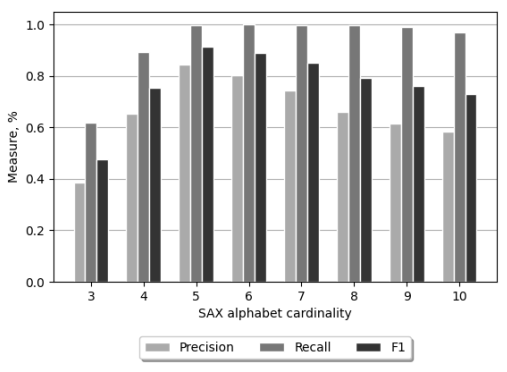

(a) UHP

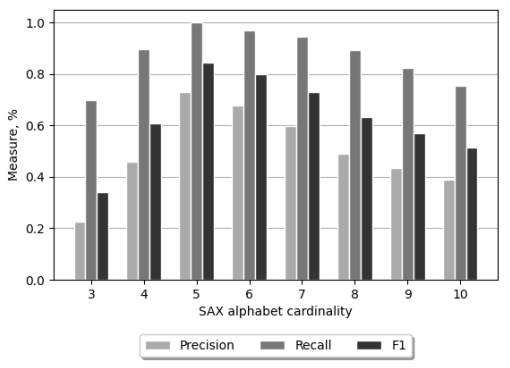

(b) DA

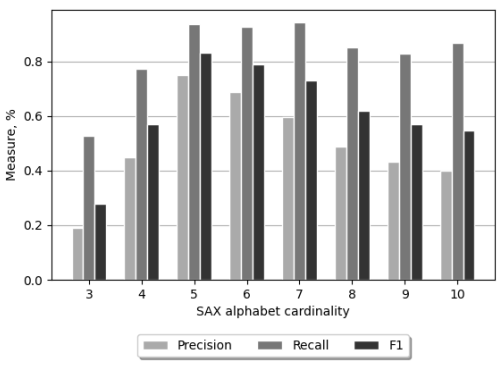

(c) FL

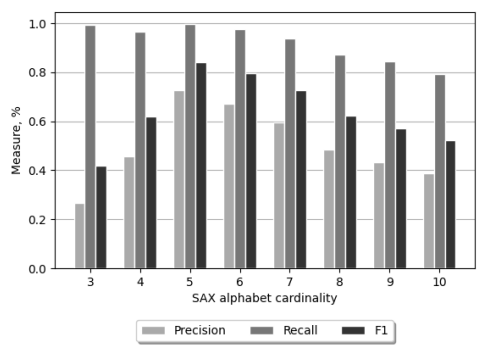

(d) UA

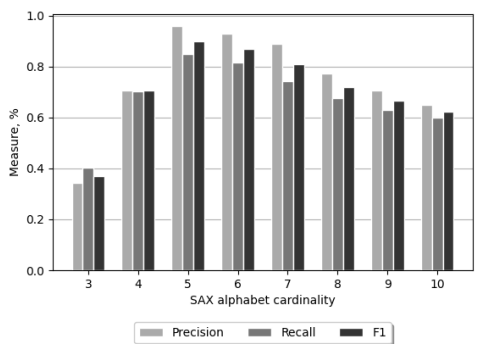

(e) DHP

Figure 6: The performance measurements for the recognition of five different shapeoids in SCOTTY.

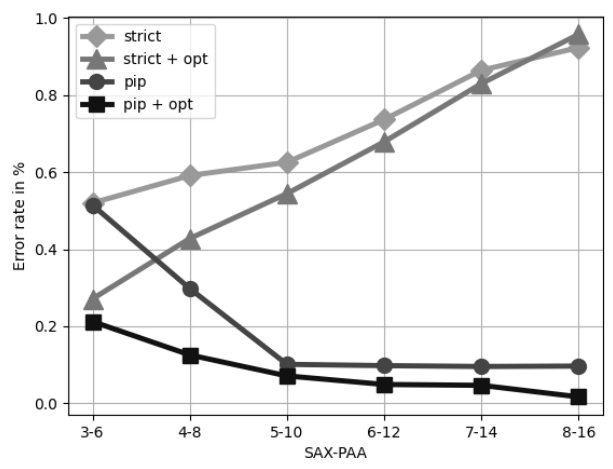

Figure 7: Classification Error Rate (in \%) using different runs with the parameter schema: $(\mathrm{SAX})-(\mathrm{PAA}=2 \mathrm{x} \mathrm{SAX})$.

relevant applications from the area of visual query systems. The advantage of the shapeoids is their ability to express more complex intentions, such as constituting a complex pattern in the time series data or having the background knowledge that the origin of the time series may contain an organisation of data points that resemble a known trendline. As future work, the shapeoids, in their current state, need to preserve all the essential features of a time series (e.g., min, max, mean), because currently SCOTTY caters only for their shape and trend type.

The evaluation of SCOTTY using the CBF dataset from the UCR Archive, uses its framework as a medium for transferring the raw-valued time series in the dataset to a

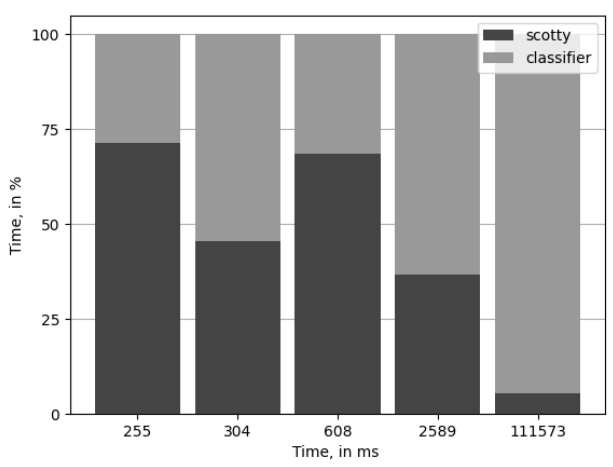

Figure 8: The overall run time ratio between the SCOTTY calculation and the pure classifier run time (ms).

declarative custom classifier. We compared its efficiency to detect the shapeoids in the CBF class definitions and the overall classification task, comparing its accuracy to stateof-the-art classifiers. The results were more than positive, especially for the overall classification run time. Another aspect worth persuing is to make ScOTTY adaptable and dynamic to streaming multivariate data. SCOTTY in its current form is limited by SAX; its interim time series representation and discritisation method. SAX normalises the time series internally in a single step, therefore requires only bounded data. It is worth to explore big data processing platforms (e. g., Apache Beam), which offer out of the box features like windowing and group-by operations for streaming data. 


\section{ACKNOWLEDGEMENT}

The research leading to these results has received partial funding from Germany's Federal Ministry of Education and Research (BMBF) under HorME.

\section{REFERENCES}

[1] P. Esling and C. Agon, "Time-series data mining," ACM Computing Surveys (CSUR), vol. 45, no. 1, pp. 1-34, 2012.

[2] T. Siddiqui, P. Luh, Z. Wang, K. Karahalios, and A. Parameswaran, "Shapesearch: A flexible and efficient system for shape-based exploration of trendlines," in Proceedings of the 2020 ACM SIGMOD International Conference on Management of Data, 2020, pp. 51-65.

[3] T. N. Bulkowski, Encyclopedia of chart patterns. John Wiley \& Sons, 2021.

[4] R. A. Wagner, R. Tabibiazar, A. Liao, and T. Quertermous, "Genomewide expression dynamics during mouse embryonic development reveal similarities to drosophila development," Developmental biology, vol. 288, no. 2, pp. 595-611, 2005.

[5] S. Ghosh, M. Feng, H. Nguyen, and J. Li, "Hypotension risk prediction via sequential contrast patterns of icu blood pressure," IEEE journal of biomedical and health informatics, vol. 20, no. 5, pp. 1416-1426, 2015.

[6] T. Siddiqui, A. Kim, J. Lee, K. Karahalios, and A. Parameswaran, "Effortless data exploration with zenvisage: an expressive and interactive visual analytics system," arXiv preprint arXiv:1604.03583, 2016.

[7] M. Mannino and A. Abouzied, "Expressive time series querying with hand-drawn scale-free sketches," in Proceedings of the $2018 \mathrm{CHI}$ Conference on Human Factors in Computing Systems, 2018, pp. 1-13.

[8] T.-c. Fu, "A review on time series data mining," Engineering Applications of Artificial Intelligence, vol. 24, no. 1, pp. 164-181, 2011.

[9] B. Hu, Y. Chen, and E. Keogh, "Time series classification under more realistic assumptions," in Proceedings of the 2013 SIAM international conference on data mining. SIAM, 2013, pp. 578-586.

[10] G. Das, K.-I. Lin, H. Mannila, G. Renganathan, and P. Smyth, "Rule discovery from time series." in $K D D$, vol. 98 , no. 1, 1998, pp. 16-22.

[11] R. Agrawal, G. Psaila, E. L. Wimmers, and M. Zaït, "Querying shapes of histories," in Proceedings of the 21th International Conference on Very Large Data Bases, 1995, pp. 502-514.

[12] B. R. Bakshi and G. Stephanopoulos, "Reasoning in time: Modeling, analysis, and pattern recognition of temporal process trends," Advances in Chemical Engineering, vol. 22, no. C, pp. 485-548, 1995.

[13] J. Lin, E. Keogh, L. Wei, and S. Lonardi, "Experiencing sax: a novel symbolic representation of time series," Data Mining and knowledge discovery, vol. 15, no. 2, pp. 107-144, 2007.

[14] A. Tsitsipas and L. Schubert, "Modelling and reasoning for indirect sensing over discrete-time via markov logic networks," in Twelfth International Workshop Modelling and Reasoning in Context, 2021.

[15] R. Agrawal, C. Faloutsos, and A. Swami, "Efficient similarity search in sequence databases," in International conference on foundations of data organization and algorithms. Springer, 1993, pp. 69-84.

[16] P. Schäfer, "Scalable time series similarity search for data analytics," Ph.D. dissertation, Humboldt-Universität zu Berlin, MathematischNaturwissenschaftliche Fakultät, 2015.

[17] C. Faloutsos, M. Ranganathan, and Y. Manolopoulos, "Fast subsequence matching in time-series databases," ACM Sigmod Record, vol. 23, no. 2 , pp. 419-429, 1994.

[18] I. Popivanov and R. J. Miller, "Similarity search over time-series data using wavelets," in Proceedings 18th international conference on data engineering. IEEE, 2002, pp. 212-221.

[19] E. J. Keogh and M. J. Pazzani, "A simple dimensionality reduction technique for fast similarity search in large time series databases," in Pacific-Asia conference on knowledge discovery and data mining. Springer, 2000, pp. 122-133.

[20] K. Chakrabarti, E. Keogh, S. Mehrotra, and M. Pazzani, "Locally adaptive dimensionality reduction for indexing large time series databases," ACM Transactions on Database Systems (TODS), vol. 27, no. 2, pp. 188-228, 2002.

[21] A. Camerra, T. Palpanas, J. Shieh, and E. Keogh, "isax 2.0: Indexing and mining one billion time series," in 2010 IEEE International Conference on Data Mining. IEEE, 2010, pp. 58-67.
[22] J. Lin, E. Keogh, S. Lonardi, and B. Chiu, "A symbolic representation of time series, with implications for streaming algorithms," in Proceedings of the 8th ACM SIGMOD workshop on Research issues in data mining and knowledge discovery, 2003, pp. 2-11.

[23] P. Schäfer and M. Högqvist, "Sfa: a symbolic fourier approximation and index for similarity search in high dimensional datasets," in Proceedings of the 15th international conference on extending database technology, 2012, pp. 516-527.

[24] T. Le Nguyen, S. Gsponer, I. Ilie, M. O'Reilly, and G. Ifrim, "Interpretable time series classification using linear models and multiresolution multi-domain symbolic representations," Data mining and knowledge discovery, vol. 33, no. 4, pp. 1183-1222, 2019.

[25] M. Butler and D. Kazakov, "Sax discretization does not guarantee equiprobable symbols," IEEE Transactions on Knowledge and Data Engineering, vol. 27, no. 4, pp. 1162-1166, 2014.

[26] B. Lkhagva, Y. Suzuki, and K. Kawagoe, "New time series data representation esax for financial applications," in 22nd International Conference on Data Engineering Workshops (ICDEW'06). IEEE, 2006, pp. x115-x115.

[27] B. Esmael, A. Arnaout, R. K. Fruhwirth, and G. Thonhauser, "Multivariate time series classification by combining trend-based and value-based approximations," in International Conference on Computational Science and Its Applications. Springer, 2012, pp. 392-403.

[28] C. T. Zan and H. Yamana, "An improved symbolic aggregate approximation distance measure based on its statistical features," in Proceedings of the 18th International Conference on Information Integration and Web-based Applications and Services, 2016, pp. 72-80.

[29] K. Song, M. Ryu, and K. Lee, "Transitional sax representation for knowledge discovery for time series," Applied Sciences, vol. 10, no. 19, p. 6980, 2020.

[30] J. Lin, R. Khade, and Y. Li, "Rotation-invariant similarity in time series using bag-of-patterns representation," Journal of Intelligent Information Systems, vol. 39, no. 2, pp. 287-315, 2012.

[31] L. Ye and E. Keogh, "Time series shapelets: a new primitive for data mining," in Proceedings of the 15th ACM SIGKDD international conference on Knowledge discovery and data mining, 2009, pp. 947956.

[32] E. Keogh, J. Lin, and A. Fu, "Hot sax: Efficiently finding the most unusual time series subsequence," in Fifth IEEE International Conference on Data Mining (ICDM'05). Ieee, 2005, pp. 8-pp.

[33] P. Senin and S. Malinchik, "Sax-vsm: Interpretable time series classification using sax and vector space model," in 2013 IEEE 13th international conference on data mining. IEEE, 2013, pp. 1175-1180.

[34] Y. Zheng, Y.-W. Si, and R. Wong, "Feature extraction for chart pattern classification in financial time series," Knowledge and Information Systems, pp. 1-42, 2021.

[35] T.-c. Fu, F.-1. Chung, R. Luk, and C.-m. Ng, "Stock time series pattern matching: Template-based vs. rule-based approaches," Engineering Applications of Artificial Intelligence, vol. 20, no. 3, pp. 347-364, 2007.

[36] G. Li, L. Zhang, and L. Yang, "Tsx: A novel symbolic representation for financial time series," in Pacific Rim International Conference on Artificial Intelligence. Springer, 2012, pp. 262-273.

[37] W. Xue, Q. Luo, and H. Wu, "Pattern-based event detection in sensor networks," Distributed and Parallel Databases, vol. 30, no. 1, pp. 27-62, 2012.

[38] J. A. Lara, D. Lizcano, A. Pérez, and J. P. Valente, "A general framework for time series data mining based on event analysis: Application to the medical domains of electroencephalography and stabilometry," Journal of biomedical informatics, vol. 51, pp. 219-241, 2014.

[39] A. Tsitsipas and L. Schubert, "On group theory and interpretable time series primitives," in Proceedings of the Seventeenth International Conference on Advanced Data Mining and Applications. Springer, 2021.

[40] F. L. K. Chung, T.-C. Fu, W. P. R. Luk, and V. T. Y. Ng, "Flexible time series pattern matching based on perceptually important points," in Workshop on Learning from Temporal and Spatial Data in International Joint Conference on Artificial Intelligence, 2001.

[41] P. Schäfer, "The boss is concerned with time series classification in the presence of noise," Data Mining and Knowledge Discovery, vol. 29 , no. 6, pp. 1505-1530, 2015.

[42] M. Wistuba, J. Grabocka, and L. Schmidt-Thieme, "Ultra-fast shapelets for time series classification," arXiv preprint arXiv:1503.05018, 2015.

[43] P. Schäfer, "Scalable time series classification," Data Mining and Knowledge Discovery, vol. 30, no. 5, pp. 1273-1298, 2016. 